\title{
Quasi-optimality of event-based encoders
}

\author{
Justin Pearson, João P. Hespanha, Daniel Liberzon
}

\begin{abstract}
We present an event-triggered controller for stabilizing a continuous-time linear time-invariant system subject to communication constraints. We model the communication constraints as a noiseless finite-capacity communication channel between the process sensors and the controller/actuator. An encoder converts the process state into symbols to send across the channel to the controller/actuator, which converts the symbols into a state estimate to be used in a simple emulation-based state-feedback control law. We derive a sufficient condition for this scheme to stabilize the process. The condition depends on the encoder's average bit-rate, its average consumption of communication resources, and the eigenvalues of the process. The proposed encoding scheme is order-optimal in the sense that its stability condition is within a constant factor of the optimal bound from previous work.
\end{abstract}

\section{INTRODUCTION}

We consider the problem of stabilizing a continuoustime linear time-invariant process subject to communication constraints. The basic setup, also considered in [16] and many other works, assumes that a finite capacity communication channel connects the process sensors to the controller/actuator. An encoder at the sensor sends a symbol through the channel once per sampling time, and the controller determines the actuation signal based on the incoming stream of symbols. The issue of efficient symbol transmission has been enthusiastically explored - see the above papers, [7, 8], and references therein.

The notion of using event-triggered control schemes to conserve communication resources is not new. Recent results [9-12] indicate that an encoder can conserve communication resources by transmitting only on a "need-to-know" basis. Motivating the present work is the observation that the absence of an explicitly transmitted symbol nevertheless conveys information. To capture this, [13] presented a framework wherein each symbol's transmission costs one unit of communication resources, except for one special free symbol that represents the absence of a transmission. The main result of [13] was an extension of the necessary and sufficient condition from [2-4] for the existence of a stabilizing controller and encoder/decoder pair obeying a constraint on its average cost per symbol.

The encoding scheme from [13] is optimal in the sense that it could be used to stabilize a process with an average cost-

J. Pearson and J. P. Hespanha are with the Department of Electrical and Computer Engineering, University of California, Santa Barbara, U.S.A., \{jppearson, hespanha\} @ece.ucsb.edu. This material is based upon work supported by the Institute for Collaborative Biotechnologies through grant W911NF-09-D-0001 from the U.S. Army Research Office.

D. Liberzon is with the Coordinated Science Laboratory, University of Illinois at Urbana-Champaign, U.S.A., Iiberzon@uiuc .edu. Supported by NSF grants CNS-1217811 and ECCS-1231196. per-symbol as low as possible. However, the scheme is possibly very complex and difficult to implement. Specifically, as an encoder's resource consumption approaches the bound of the stability condition, the size of its codeword library grows to infinity, requiring infinite memory and computational power. In this paper, we address this by developing an easilyimplementable event-based encoder/decoder and compare it to the encoders from [13]. Specifically, the main technical contribution of this paper is a sufficient condition on the average bit-rate, process eigenvalues, and average cost-persymbol for the existence of a stabilizing emulation-based controller and event-based encoder/decoder pair. We then establish that the proposed event-based encoding scheme is "order-optimal" in the sense that the sufficiency bound is within a constant factor of the necessary and sufficient bound from [13].

Preliminary work in event-based control assumed that the event-detector could transmit infinite-precision quantities across the communication channel to the controller/actuator. To extend this work to finite-bit-rate communication channels, recent works explore event-based quantized control, typically introducing an encoder/decoder or quantizer in the communication path to limit the number of bits transmitted. Several recent works offer strategies for event-based quantized control that study trade-offs between quantizer complexity, bit-rate, and minimum inter-transmission intervals. For example, [14] explores an intuitive event-based quantized control scheme that sends single bits based on the state estimation error transitioning between quantization levels. The design in [15] of an event-based quantized control scheme for a disturbed, stable LTI system allows the state trajectory to match as closely as desired the statefeedback state trajectory that would be obtained without communication constraints. In [16] the authors consider the simultaneous co-design of the event-generator and quantizer for control of a non-linear system using the hybrid system framework from [17]. Sufficient bit-rates for event-triggered stabilizability of nonlinear systems were also studied in [18]. In [19] a method is developed for event-based quantized control design that achieves a desired convergence rate of a Lyapunov function of the state while guaranteeing a positive lower bound on inter-transmission times and a uniform upper bound on the number of bits in each transmission.

The remainder of this paper is organized as follows. In Section II we set up the problem. We then describe the emulation-based controller and event-based encoder/decoder pair in Section III and prove that it stabilizes the system provided a sufficient condition holds. We summarize the work in Section IV. 


\section{Problem Statement}

In this paper we consider a stabilizable linear timeinvariant process

$$
\dot{x}=A x+B u, \quad x \in \mathbb{R}^{n}, u \in \mathbb{R}^{m},
$$

for which it is known that $x(0)$ belongs to a known bounded set $\mathcal{X}_{0} \subset \mathbb{R}^{n}$. A sensor that measures the state $x(t)$ is connected to the actuator through a finite-data-rate, errorfree, and delay-free communication channel. An encoder collocated with the sensor samples the state at a fixed sequence of transmission times $\left\{t_{k} \in[0, \infty): k \in \mathbb{N}_{>0}\right\}$, and from this sequence of measurements $\left\{x\left(t_{k}\right): k \in \mathbb{N}_{>0}\right\}$ causally constructs a sequence of symbols $\left\{s_{k} \in \mathcal{A}: k \in \mathbb{N}_{>0}\right\}$ from a nonempty finite alphabet $\mathcal{A}$. Without loss of generality, $\mathcal{A}=\{0,1, \ldots, S\}$ with $S:=|\mathcal{A}|-1$. At time $t_{k}$ the encoder sends symbol $s_{k}$ through the channel to a decoder/controller collocated with the actuator, which causally constructs the control signal $u(t), t \geqslant 0$ from the sequence of symbols $\left\{s_{k} \in \mathcal{A}: k \in \mathbb{N}_{>0}\right\}$ that arrive at the decoder. The sequence of transmission times $\left\{t_{k}\right\}$ is assumed to be monotonically nondecreasing and unbounded (i.e., $\lim _{k \rightarrow \infty} t_{k}=+\infty$ ). The fact that the sequence of transmission times is fixed a priori prevents the controller from communicating information in the transmission times themselves. Note that because the sequence of transmission times is not necessarily strictly increasing, this allows multiple transmissions at a single time instant, which can be viewed as encoding several symbols in the same message.

The non-negative average bit-rate $r$ of a sequence of symbols $\left\{s_{k}\right\} \subset\{0, \ldots, S\}$ transmitted at times $\left\{t_{k}\right\}$ is the rate of transmitted information in units of bits per time unit, and is defined as

$$
r:=\log _{2}(S+1) \limsup _{k \rightarrow \infty} \frac{k}{t_{k}} .
$$

We assume that the symbol $0 \in \mathcal{A}$ can be transmitted without consuming any communication resources, but the other $S$ symbols each require one unit of communication resources per transmission. One can think of the "free" symbol 0 as the absence of an explicit transmission. The "communication resources" at stake may be energy, time, or any other resource that may be consumed in the course of the communication process. In order to capture the average rate at which an encoder consumes communication resources, we define the average cost per symbol of an encoder as follows: We say an encoder has average cost per symbol not exceeding $\gamma$ if there exists a non-negative integer $N_{0}$ such that for every symbol sequence $\left\{s_{k}\right\}$ generated by the encoder, we have

$$
\frac{1}{N_{2}} \sum_{k=N_{1}}^{N_{1}+N_{2}-1} I_{s_{k} \neq 0} \leqslant \gamma+\frac{N_{0}}{N_{2}} \quad \forall N_{1}, N_{2} \in \mathbb{N}_{>0},
$$

where $I_{s_{k} \neq 0}:=1$ if the $k$ th symbol is not the free symbol, and 0 if it is. The summation in (3) captures the total resources spent transmitting symbols $s_{N_{1}}, s_{N_{1}+1}, \ldots, s_{N_{1}+N_{2}-1}$. Motivating this definition of average cost per symbol is the observation that the lefthand side has the intuitive interpretation of the average cost per transmitted symbol between symbols $s_{N_{1}}$ and $s_{N_{1}+N_{2}-1}$. As $N_{2} \rightarrow \infty$, the rightmost term vanishes, leaving $\gamma$ as an upper bound on the average long-term cost per symbol of the symbol sequence. Note that the average cost per symbol $\gamma$ of any encoder always satisfies $\gamma \in[0,1]$ and does not depend on the transmission times $\left\{t_{k}\right\}$.

The basic problem at hand is to find an encoder/decoder pair and controller that stabilize the state of the process while using as few communication resources as possible - small average bit-rate $r$ and average cost per symbol $\gamma$. The next section proposes a scheme and proves a condition on $r$ and $\gamma$ sufficient for stabilization.

\section{EVENT-DRIVEN ENCODERS}

This section presents results for a so-called emulationbased controller with an event-based encoder/decoder pair. We first introduce these two concepts, then we present the main result - a sufficient condition on the channel average bit-rate $r$, the encoder/decoder pair's average cost per symbol $\gamma$, and the process eigenvalues $\lambda_{i}[A]$ to ensure the existence of an emulation-based controller and eventbased encoder/decoder pair obeying the average bit-rate and average cost per symbol constraints, which together bound the state of the process. The proof is constructive in that we provide the controller, encoder, and decoder. The proposed scheme is similar to the distributed-sensor scheme of [20], in which each coordinate of a plant measurement is distributed to a dedicated encoder.

The controller is called emulation-based because it emulates a stabilizing state-feedback controller $u:=K x$. This state-feedback controller cannot be used in the limitedcommunication environment considered in this paper because the infinite-precision state $x(t) \in \mathbb{R}^{n}$ cannot be sent over the channel and hence is unavailable to the controller. Instead, in emulation-based control, the state-feedback controller is coupled to an encoder/decoder pair that estimates the state as $\hat{x}(t)$, resulting in the control law $u(t)=K \hat{x}(t), t \geqslant 0$.

The encoder/decoder pair is called event-based because the encoder sends non-free symbols only in the event that the process state satisfies a particular condition. In the proposed scheme, the particular condition is that the magnitude of any component of the state estimation error grows beyond a predetermined fixed size. Provided the error stays within the prescribed box, the encoder sends only the free symbol and thus consumes no communication resources. This justifies the label "event-based."

We now describe the controller and encoder/decoder pair. The controller is an emulation-based controller $u=K \hat{x}$, where $A+B K$ is Hurwitz. The encoder and decoder rely on the following lemma, proved in the appendix, which establishes a useful coordinate transformation.

Lemma 1: Assume $A$ is diagonalizable over $\mathbb{C}$. Consider the process and the estimated process

$$
\begin{aligned}
& \dot{x}(t)=A x(t)+B u(t), \quad x\left(t_{0}\right)=x_{0} \quad t \in\left[t_{0}, t_{1}\right) \\
& \dot{\hat{x}}(t)=A \hat{x}(t)+B u(t), \quad \hat{x}\left(t_{0}\right)=\hat{x}_{0} \quad t \in\left[t_{0}, t_{1}\right) \text {. }
\end{aligned}
$$


There exists a time-varying matrix $P(t) \in \mathbb{R}^{n \times n}$ such that for any $t_{0}, t_{1}, x_{0}, \hat{x}_{0}$, the state estimation error

$$
e(t):=P(t)(x(t)-\hat{x}(t))
$$

satisfies

$$
\dot{e}_{i}(t)=a_{i} e_{i}(t), \quad i=1, \ldots, n, \quad t \in\left[t_{0}, t_{1}\right)
$$

where the $a_{i}$ are the real parts of the eigenvalues of $A$. Moreover, there exists a positive $\epsilon$ for which

$$
\sigma_{\min }(P(t)) \geqslant \epsilon \quad t \geqslant 0,
$$

where $\sigma_{\min }(\cdot)$ denotes the smallest singular value.

The encoder and decoder each run internal copies of the process as described by (5), with $\hat{x}(0):=0$. Lemma 1 establishes that the state estimation error $e(t)$ evolves like $n$ decoupled scalar linear systems (7) during the time intervals between instantaneous updates to the state estimate. Define

$$
L_{i}:=\sup _{x_{0} \in \mathcal{X}_{0}}\left|\left(P(0) x_{0}\right)_{i}\right| \quad i=1, \ldots, n
$$

so that we have $e_{i}(0) \in\left[-L_{i}, L_{i}\right]$ for $i=1, \ldots, n$. The basic idea is to monitor in parallel each of these one-dimensional subsystems and as long as it stays inside a fixed interval, send the free symbol 0 . A non-free symbol is sent only when the one-dimensional error leaves the $\left[-L_{i}, L_{i}\right]$ interval: send -1 if the error exits the left side of the interval and send 1 if it exits out the right side. To this end, the encoder contains $n$ sub-encoders, where sub-encoder $i$ monitors $e_{i}(t)$. Partition the systems (7) based on whether or not they are stable:

$$
\begin{aligned}
& \mathcal{I}_{1}:=\left\{i \in\{1, \ldots, n\}: a_{i}<0\right\} \\
& \mathcal{I}_{2}:=\left\{i \in\{1, \ldots, n\}: a_{i} \geqslant 0\right\} .
\end{aligned}
$$

Scalar systems in $\mathcal{I}_{1}$ are stable, so we will soon see that there is no need to transmit information on their behalf. On the other hand, for $i \in \mathcal{I}_{2}$, at time $k T_{i}, k \in \mathbb{N}_{>0}$ (with $T_{i}$ to be determined shortly), sub-encoder $i$ sends symbol $s_{i, k} \in\{-1,0,1\}$ according to

$$
s_{i, k}= \begin{cases}-1 & e_{i}\left(k T_{i}\right)<-L_{i} \\ 0 & e_{i}\left(k T_{i}\right) \in\left[-L_{i}, L_{i}\right] \quad k \in \mathbb{N}_{>0} . \\ 1 & e_{i}\left(k T_{i}\right)>L_{i}\end{cases}
$$

Note that the $i$ th sub-encoder consumes no communication resources if $e_{i}\left(k T_{i}\right) \in\left[-L_{i}, L_{i}\right]$. Immediately after a transmission, the encoder and decoder then each update their state estimates as

$$
\hat{x}\left(k T_{i}\right)^{+}=\hat{x}\left(k T_{i}\right)^{-}+P\left(k T_{i}\right)^{-1} \mathbf{v}_{i} \Delta_{i}\left(s_{i, k}\right),
$$

where $P(t)$ is from Lemma $1, \mathbf{v}_{i} \in \mathbb{R}^{n}$ is the $i$ th unit vector, and for each dimension $i$, the decoding function $\Delta_{i}:\{-1,0,1\} \rightarrow \mathbb{R}$ is defined as

$$
\Delta_{i}(s):=\left\{\begin{array}{cl}
-\frac{L_{i}}{2}\left(1+\exp \left(a_{i} T_{i}\right)\right) & s=-1 \\
0 & s=0 \\
\frac{L_{i}}{2}\left(1+\exp \left(a_{i} T_{i}\right)\right) & s=1 .
\end{array}\right.
$$

Note that the values of $\Delta_{i}$ are merely the midpoints of the intervals $\left[L_{i}, L_{i} \exp \left(a_{i} T_{i}\right)\right]$ and $\left[-L_{i},-L_{i} \exp \left(a_{i} T_{i}\right)\right]$.
It is worth mentioning here that the encoder's transmission times are entirely fixed and determined a priori, consistent with the problem statement. The event-based nature of this scheme lies in whether or not a particular transmission consumes communication resources.

This concludes the description of the event-based encoder/decoder pair, except for the precise choice of the transmission periods $T_{i}$. The following theorem states a condition under which one can choose transmission periods $T_{i}$ that bound the state while obeying certain communication constraints.

Define the monotonically increasing continuous function $h:[0, \ln 3) \rightarrow[0, \infty)$ as

$$
h(x):=\frac{x}{\ln \frac{2}{e^{x}-1}} .
$$

Theorem 1: Consider the process (1), and assume that $A$ is diagonalizable over $\mathbb{C}$ and $A+B K$ is Hurwitz. For every $\gamma \in[0,1]$ and $r>0$ satisfying

$$
r \frac{h^{-1}(\gamma)}{\ln 3} \ln 2 \geqslant \sum_{i: \Re \lambda_{i}[A] \geqslant 0} \lambda_{i}[A],
$$

there exists an emulation-based controller and event-based encoder/decoder pair of the type described above that keeps the state of the process bounded for every initial condition in $\mathcal{X}_{0}$; the encoder has average bit-rate not exceeding $r$ and has average cost per symbol not exceeding $\gamma$.

Remark 1: For the special case of $n=1$ (scalar system) and $\gamma=1$ (no power constraint), this event-based encoding scheme selects the transmission period $T:=h^{-1}(1) / \lambda=$ $\ln 2 / \lambda$ and hence bounds the state estimation error within the interval $[-2 L, 2 L]$.

Remark 2: In [13] the authors proved a necessary and sufficient condition on the average bit-rate $r$ and average cost per symbol $\gamma$ for the existence of a stabilizing controller and encoder/decoder pair:

$$
r f(\gamma, S) \ln 2 \geqslant \sum_{i: \Re \lambda_{i}[A] \geqslant 0} \lambda_{i}[A],
$$

where the function $f:[0,1] \times[0, \infty) \rightarrow[0, \infty)$ is defined as

$$
f(\gamma, S):= \begin{cases}\frac{H(\gamma)+\gamma \log _{2} S}{\log _{2}(S+1)} & 0 \leqslant \gamma \leqslant \frac{S}{S+1} \\ 1 & \frac{S}{S+1}<\gamma \leqslant 1,\end{cases}
$$

and $H(p):=-p \log _{2}(p)-(1-p) \log _{2}(1-p)$ is the base2 entropy of a Bernoulli random variable with parameter $p$. The function $f(\gamma, S)$ is nonincreasing in $S$. Whereas the necessary and sufficient bounds from [13] had the term $f(\gamma, S)$, the event-based encoding bound in (14) has the term $h^{-1}(\gamma) / \ln 3$. The ratio

$$
g(\gamma, S):=\frac{f(\gamma, S)}{\frac{h^{-1}(\gamma)}{\ln 3}}
$$

captures the factor by which the event-based bound exceeds the theoretical bound developed in the previous sections. We numerically observe: 
- $g(\gamma, 1)<2.43$ for all $\gamma \in(0,1]$. This demonstrates that this encoding and control scheme is never more than 2.43 times more conservative than the optimal bound established in [13]. Specifically, if a given process may be bounded with a certain average bit-rate $r$, then there exists a average bit-rate $\tilde{r}$ not exceeding $2.43 r$ such that this event-based scheme can bound the process using average bit-rate $\tilde{r}$.

- $g(1, S) \leqslant \ln 3 / \ln 2 \approx 1.58$ for all $S \in \mathbb{N}_{>0}$. This states that without the average cost per symbol constraint $(\gamma \rightarrow 1)$, this event-based encoding scheme is 1.58 times more conservative than the optimal average bitrate bound from [13].

\section{A. Proof of Theorem 1}

The main idea of the proof is to show that when assumption (14) holds, it is possible to allocate the available average bit-rate among each of the $n$ sub-encoders in such a way that each sub-encoder has a sufficiently large average bit-rate to bound its component of the state estimation error.

For sub-encoder $i \in \mathcal{I}_{2}$, we pick the transmission period $T_{i}$ as

$$
T_{i}:=\frac{h^{-1}(\gamma)}{a_{i}}
$$

where the definition of $h$ is from (13). As mentioned above, no information need be sent on behalf of the stable systems $i \in \mathcal{I}_{1}$.

1) The scheme's average bit-rate does not exceed $r$ : For $i \in \mathcal{I}_{2}$, sub-encoder $i$ sends one of 3 symbols every $T_{i}$ time units, resulting in an average bit-rate of

$$
r_{i}:=\frac{\log _{2} 3}{T_{i}},
$$

and so the average bit-rate used by the encoder as a whole is simply

$$
\begin{aligned}
\sum_{i \in \mathcal{I}_{2}} r_{i} & =\log _{2} 3 \sum_{i \in \mathcal{I}_{2}} \frac{1}{T_{i}} \\
& =\frac{\log _{2} 3}{h^{-1}(\gamma)} \sum_{i \in \mathcal{I}_{2}} a_{i} \\
& =\frac{\log _{2} 3}{h^{-1}(\gamma)} \sum_{i: \Re \lambda_{i}[A] \geqslant 0} \lambda_{i}[A] \\
& \leqslant r,
\end{aligned}
$$

where the last inequality follows from hypothesis (14). Hence, this encoding scheme uses a average bit-rate of $r$ or less.

2) The scheme bounds the state of the process: Next we show that this controller and event-based encoder/decoder pair bound the state of the process (1). From the process (1) and the definition of $e(t)$, the state evolves as

$$
\dot{x}(t)=(A+B K) x(t)-B K P(t)^{-1} e(t) .
$$

Since $(A+B K)$ is Hurwitz, then the state $x(t)$ is bounded provided that $e(t)$ is bounded and $P(t)^{-1}$ has bounded maximum singular value. The latter is ensured because Lemma 1 guarantees a positive $\epsilon$ for which $\sigma_{\min }(P(t)) \geqslant \epsilon>0$ for all $t$. The remainder of the proof shows that $e(t)$ is bounded under the proposed scheme. Since $e_{i}(t) \rightarrow 0$ for $i \in \mathcal{I}_{1}$, we focus on $e_{i}(t)$ for $i \in \mathcal{I}_{2}$.

We proceed with an inductive proof that sequence $\left\{e_{i}\left(k T_{i}\right)\right\}_{k \in \mathbb{N}_{>0}}$ is bounded for $i \in \mathcal{I}_{2}$. The base case $k=$ 0 follows from the definition of $L_{i}$. Next we prove that $e_{i}\left(k T_{i}\right) \in\left[-L_{i}, L_{i}\right]$ provided that $e_{i}\left(k T_{i}-T_{i}\right) \in\left[-L_{i}, L_{i}\right]$. If $e_{i}\left(k T_{i}-T_{i}\right)$ is so small that it does not grow outside the box $\left[-L_{i}, L_{i}\right]$ by the next timestep, then we naturally have $e_{i}\left(k T_{i}\right) \in\left[-L_{i}, L_{i}\right]$. On the other hand, suppose at specific time $t^{*}$ satisfying $k T_{i}-T_{i} \leqslant t^{*}<k T_{i}$, the scalar error $e_{i}\left(t^{*}\right)$ grows to the boundary of the box $\left[-L_{i}, L_{i}\right]$; without loss of generality suppose $e_{i}\left(t^{*}\right)=L_{i}$. Up to $T_{i}$ time units later, timestep $k T_{i}$ occurs and sub-encoder $i$ transmits $s_{i, k}=1$ to the decoder. Upon receiving symbol 1 , the decoder knows from the encoding scheme (10) that the scalar error $e_{i}\left(k T_{i}\right)^{-}$immediately before the transmission lies in the interval

$$
e_{i}\left(k T_{i}\right)^{-} \in\left[L_{i}, L_{i} e^{a_{i} T_{i}}\right]
$$

We can express the scalar error $e_{i}\left(k T_{i}\right)^{-}$as the vector $e\left(k T_{i}\right)^{-}$times $i$ th unit vector

$$
\begin{aligned}
e_{i}\left(k T_{i}\right)^{-} & =\mathbf{v}_{i}^{\prime} e\left(k T_{i}\right)^{-} \\
& =\mathbf{v}_{i}^{\prime} P\left(k T_{i}\right)\left(x\left(k T_{i}\right)^{-}-\hat{x}\left(k T_{i}\right)^{-}\right) .
\end{aligned}
$$

Rearranging the update rule (11) yields an expression for $\hat{x}\left(k T_{i}\right)^{-}$:

$$
\hat{x}\left(k T_{i}\right)^{-}=\hat{x}\left(k T_{i}\right)^{+}-P\left(k T_{i}\right)^{-1} \mathbf{v}_{i} \Delta_{i}(1) .
$$

Plugging this into (27) yields

$$
\begin{aligned}
e_{i}\left(k T_{i}\right)^{-}= & \mathbf{v}_{i}^{\prime} P\left(k T_{i}\right)\left(x\left(k T_{i}\right)^{-}-\hat{x}\left(k T_{i}\right)^{+}\right. \\
& \left.+P\left(k T_{i}\right)^{-1} \mathbf{v}_{i} \Delta_{i}(1)\right) \\
= & \mathbf{v}_{i}^{\prime} P\left(k T_{i}\right)\left(x\left(k T_{i}\right)^{-}-\hat{x}\left(k T_{i}\right)^{+}\right) \\
& \quad+\Delta_{i}(1) \\
= & e_{i}\left(k T_{i}\right)^{+}+\Delta_{i}(1),
\end{aligned}
$$

where we used the fact that $x\left(k T_{i}\right)^{-}=x\left(k T_{i}\right)^{+}$due to the continuity of the solution $x(t)$. Plugging this into (25) yields

$$
\begin{array}{rlrl}
e_{i}\left(k T_{i}\right)^{+}+\Delta_{i}(1) & \in\left[L_{i}, L_{i} e^{a_{i} T_{i}}\right] \\
\Leftrightarrow & e_{i}\left(k T_{i}\right)^{+} & \in\left[-L_{i}, L_{i}\right]\left(e^{a_{i} T_{i}}-1\right) / 2 .
\end{array}
$$

Recall that $T_{i}$ was chosen to satisfy $h\left(a_{i} T_{i}\right)=\gamma \in[0,1]$. Applying $h^{-1}$ to this inclusion yields $a_{i} T_{i} \in[0, \ln 2]$, and therefore $\left(e^{a_{i} T_{i}}-1\right) / 2 \leqslant 1$. Applying this to (33) establishes that $e_{i}\left(k T_{i}\right) \in\left[-L_{i}, L_{i}\right]$ and completes the inductive proof that the sequence $\left\{e_{i}\left(k T_{i}\right)\right\}_{k \in \mathbb{N}_{>0}}$ is bounded. Since $e_{i}(t)$ grows exponentially between transmission times, we conclude that $e_{i}(t)$ is bounded as well. Since this holds for all $i \in \mathcal{I}_{2}$ and $e_{j}(t) \rightarrow 0$ for $j \in \mathcal{I}_{1}$, this controller and encoder/decoder pair bound the estimation error. Therefore the state is bounded for all time as well. 
3) The scheme's average cost does not exceed $\gamma$ : Lastly we prove that this encoding scheme has average cost per symbol not exceeding $\gamma$. The symbol stream emitted by the encoder is comprised of the simultaneous individual symbol sequences $\left\{s_{i, k}\right\}_{k \in \mathbb{N}_{>0}}, i \in \mathcal{I}_{2}$. We first show that each individual symbol sequence has average cost per symbol not exceeding $\gamma$. Then we show that superimposing these sequences preserves this property.

Consider sub-encoder $i \in \mathcal{I}_{2}$. If $\gamma=1$ then the average cost per symbol condition (3) is satisfied trivially. Next suppose $\gamma \in[0,1)$. Applying $h^{-1}$ to this inclusion yields $a_{i} T_{i} \in[0, \ln 2)$ and hence $e^{a_{i} T_{i}}-1<2$. By (33), this implies $\left|e_{i}\left(k T_{i}\right)\right|<L_{i}$ with strict inequality, so there will be a strictly positive period of time $\tau_{i}>0$ until $e_{i}(t)$ grows to leave the $\left[-L_{i}, L_{i}\right]$ box. During this time, no non-free symbols will be transmitted. The "dead time" $\tau_{i}$ is simply the amount of time required for the bound $L_{i}\left(e^{a_{i} T_{i}}-1\right) / 2$ to grow to size $L_{i}$. Lemma 1 proved that $e_{i}(t)$ grows exponentially, so $\tau_{i}$ satisfies

$$
\begin{aligned}
e^{a_{i} \tau_{i}} L_{i} & \left(e^{a_{i} T_{i}}-1\right) / 2=L_{i} \\
\Leftrightarrow \quad \tau_{i} & =\frac{1}{a_{i}} \ln \frac{2}{e^{a_{i} T_{i}}-1} \\
& =\frac{T_{i}}{h\left(a_{i} T_{i}\right)}=\frac{T_{i}}{\gamma},
\end{aligned}
$$

where (36) follows from the definitions of $h$ and $T_{i}$. This establishes a bound on the number of non-free transmissions as follows. Consider the symbol sequence $\left\{s_{i, k}\right\}_{k \in \mathbb{N}_{>0}}$ emitted by this encoding scheme. Let $N_{2}, N_{1}$ be arbitrary positive integers, and let $N_{\mathrm{nf}}:=\sum_{k=N_{1}}^{N_{1}+N_{2}-1} I_{s_{i, k} \neq 0}$ be the number of non-free symbols among symbols $s_{N_{1}}, \ldots, s_{N_{1}+N_{2}-1}$. Let $t_{j}, j=1, \ldots, N_{\mathrm{nf}}$ be the time that the $j$ th non-free transmission occurred. The $t_{j}$ satisfy $N_{1} T_{i} \leqslant t_{1}<\ldots<$ $t_{N_{\mathrm{nf}}} \leqslant\left(N_{1}+N_{2}-1\right) T_{i}$. Only free symbols are transmitted in the time interval $\left[t_{j}, t_{j}+\tau_{i}\right)$, and so

$$
t_{j} \geqslant \tau_{i}+t_{j-1}, \quad j=2, \ldots, N_{\text {nf }}
$$

Iterating this formula over $j$, we obtain

$$
t_{N_{\mathrm{nf}}} \geqslant \tau_{i}\left(N_{\mathrm{nf}}-1\right)+t_{1} .
$$

Rearranging this and using the facts that $N_{1} T_{i} \leqslant t_{1}$ and $t_{N_{\mathrm{nf}}} \leqslant\left(N_{1}+N_{2}-1\right) T_{i}$, we obtain

$$
\sum_{k=N_{1}}^{N_{1}+N_{2}-1} I_{s_{i, k} \neq 0}=: N_{\mathrm{nf}} \leqslant \frac{T_{i}}{\tau_{i}} N_{2}+1=\gamma N_{2}+1,
$$

where we leveraged (36). This satisfies the average cost per symbol condition (3), so we conclude that for any $i \in \mathcal{I}_{2}$, the symbol sequence $\left\{s_{i, k}\right\}_{k \in \mathbb{N}_{>0}}$ has average cost per symbol not exceeding $\gamma$.

Finally we show that superimposing the symbol streams results in a stream with average cost per symbol not exceeding $\gamma$. Let $N_{1}, N_{2} \in \mathbb{N}$ be arbitrary positive integers, and let $\mathcal{J}_{i}, i=1, \ldots, n$ partition $\left\{N_{1}, N_{1}+1, \ldots, N_{1}+N_{2}-1\right\}$ such that $\mathcal{J}_{i}$ is the set of indices between $N_{1}$ and $N_{1}+N_{2}-1$ where the transmitted symbol was sent by sub-encoder $i$. Then $\sum_{i=1}^{n}\left|\mathcal{J}_{i}\right|=N_{2}$, and we obtain

$$
\begin{aligned}
\sum_{k=N_{1}}^{N_{1}+N_{2}-1} I_{s_{i, k} \neq 0} & =\sum_{i=1}^{n} \sum_{k \in \mathcal{J}_{i}} I_{s_{i, k} \neq 0} \\
& \leqslant \sum_{i=1}^{n}\left(\gamma\left|\mathcal{J}_{i}\right|+N_{0, i}\right) \\
& =\gamma N_{2}+N_{0},
\end{aligned}
$$

where $N_{0}:=\sum_{i=1}^{n} N_{0, i}$. The inequality comes from leveraging (3) for each sub-encoder on its respective index interval $\mathcal{J}_{i}$. This completes the proof of Theorem 1 .

Remark 3: Theorem 1 constructs a scheme that bounds the state $x(t)$ of the process (1). We now use this result to informally derive a condition for exponential stabilization of $x(t)$. Let $\bar{x}(t):=e^{\epsilon t} x(t)$, where $\epsilon>0$ is small enough that

$$
r \frac{h^{-1}(\gamma)}{\ln 3} \ln 2>\sum_{i: \Re \lambda_{i}[A] \geqslant 0} \lambda_{i}[A]+n \epsilon,
$$

and suppose $A+\epsilon I+B K$ is Hurwitz. Applying Theorem 1 to the $\bar{x}$ system provides a controller and encoder/decoder that bounds $\bar{x}$. However,

$$
\|\bar{x}(t)\| \leqslant c \quad \Leftrightarrow \quad\|x(t)\|<c e^{-\epsilon t},
$$

and so the state converges to 0 exponentially fast.

\section{CONClusion AND Future Work}

In this paper we proposed an event-triggered control scheme for bounding the state of a continuous-time linear process under communication constraints. We considered constraints on both the channel average bit-rate and the encoding scheme's average cost per symbol.

Our scheme's requirements on communication resources are order-optimal in terms of the necessary and sufficient condition for stabilizability from previous work. This helps justify the use of event-based controllers in limitedcommunication control schemes.

A limitation of our scheme is the assumption that the encoder has access to the full state. Extensions to outputfeedback are the subject of future work.

\section{APPENDIX}

Proof of Lemma 1. Let $P(t):=Q R(t) Q^{-1}$, where $Q \in$ $\mathbb{R}^{n \times n}$ is a real invertible matrix that transforms $A$ to its real Jordan normal form, namely

$$
Q^{-1} A Q=\Lambda:=\operatorname{diag}\left(a_{1}, \ldots, a_{n_{r}}, \Lambda_{n_{r}+1}, \ldots, \Lambda_{n_{b}}\right),
$$

where $a_{1}, \ldots, a_{n_{r}} \in \mathbb{R}$ are the real eigenvalues of $A$ and each of the 2 -by- 2 matrices $\Lambda_{n_{r}+1}, \ldots, \Lambda_{n_{b}} \in \mathbb{R}^{2 \times 2}$ has the form

$$
\Lambda_{i}:=\left[\begin{array}{cc}
a_{i} & b_{i} \\
-b_{i} & a_{i}
\end{array}\right], \quad i=n_{r}+1, \ldots, n_{b},
$$

where $a_{i} \pm j b_{i}$ is a pair of complex conjugate eigenvalues of $A$. The time-varying invertible block-diagonal matrix $R(t) \in$ $\mathbb{R}^{n \times n}$ is defined for all $t \geqslant 0$ as

$$
R(t):=\operatorname{diag}\left(I_{n_{r} \times n_{r}}, R_{n_{r}+1}(t), \ldots, R_{n_{b}}(t)\right)
$$


where

$$
R_{i}(t):=\left[\begin{array}{cc}
\cos \left(b_{i} t\right) & -\sin \left(b_{i} t\right) \\
\sin \left(b_{i} t\right) & \cos \left(b_{i} t\right)
\end{array}\right], \quad i=n_{r}+1, \ldots, n_{b} .
$$

We now show that $e(t)=e^{D\left(t-t_{0}\right)} e\left(t_{0}\right)$ for $t \in\left[t_{0}, t_{1}\right)$, where $D$ is a diagonal real matrix of the real parts of the eigenvalues of $A$ :

$$
D:=\operatorname{diag}\left(a_{1}, \ldots, a_{n_{r}}, a_{n_{r}+1} I_{2 \times 2}, \ldots, a_{n_{b}} I_{2 \times 2}\right) .
$$

Using the definitions of $P(t)$ and $Q$ yields

$$
e(t)=Q R(t) Q^{-1} e^{Q \Lambda Q^{-1}\left(t-t_{0}\right)}\left(x\left(t_{0}\right)-\hat{x}\left(t_{0}\right)\right) .
$$

It is easily verified from the definition of matrix exponential that $e^{\Lambda_{i} t}=e^{a_{i} t} R_{i}^{-1}(t)$. Therefore (46) becomes

$$
\begin{aligned}
e(t) & =Q R(t) e^{D\left(t-t_{0}\right)} R\left(t-t_{0}\right)^{-1} Q^{-1}\left(x\left(t_{0}\right)-\hat{x}\left(t_{0}\right)\right) \\
& =e^{D\left(t-t_{0}\right)} P\left(t_{0}\right)\left(x\left(t_{0}\right)-\hat{x}\left(t_{0}\right)\right)=e^{D\left(t-t_{0}\right)} e\left(t_{0}\right),
\end{aligned}
$$

where we used the fact that $R(t) R\left(t-t_{0}\right)^{-1}=R\left(t_{0}\right)$. Therefore the state estimation error $e(t)$ evolves as $n$ scalar decoupled error subsystems

$$
\dot{e}_{i}(t)=a_{i} e_{i}(t), \quad i=1, \ldots, n, \quad t \in\left[t_{0}, t_{1}\right) .
$$

Lastly, it is straightforward to verify that the minimum singular value of $R_{i}(t)$ is $\sigma_{\min }\left(R_{i}(t)\right)=1$ for any $t$. Moreover, since $Q$ is invertible, there exists $\epsilon>0$ for which $\sigma_{\min }(P(t)) \geqslant \epsilon$ for all $t$. This concludes the proof.

\section{REFERENCES}

[1] R. Brockett and D. Liberzon, "Quantized feedback stabilization of linear systems," Automatic Control, IEEE Transactions on, vol. 45, no. 7, pp. 1279-1289, Jul 2000.

[2] J. P. Hespanha, A. Ortega, and L. Vasudevan, "Towards the control of linear systems with minimum bit-rate," in Proc. of the Int. Symp. on the Mathematical Theory of Networks and Syst., Aug. 2002.

[3] G. Nair and R. Evans, "Communication-limited stabilization of linear systems," in Decision and Control, 2000. Proceedings of the 39th IEEE Conference on, vol. 1, 2000, pp. $1005-1010$.

[4] S. Tatikonda and S. Mitter, "Control under communication constraints," Automatic Control, IEEE Transactions on, vol. 49, no. 7, pp. 1056 - 1068, july 2004.

[5] G. N. Nair and R. J. Evans, "Exponential stabilisability of finite-dimensional linear systems with limited data rates," Automatica, vol. 39, no. 4, pp. 585 - 593, 2003.

[6] A. Matveev and A. Savkin, "Multirate stabilization of linear multiple sensor systems via limited capacity communication channels," SIAM Journal on Control and Optimization, vol. 44, no. 2, pp. 584-617, 2005.

[7] G. Nair, "A nonstochastic information theory for communication and state estimation," Automatic Control, IEEE Transactions on, vol. 58, no. 6, pp. 1497-1510, June 2013.

[8] A. Matveev and A. Savkin, "An analogue of shannon information theory for detection and stabilization via noisy discrete communication channels," SIAM Journal on Control and Optimization, vol. 46, no. 4, pp. 1323-1367, 2007. [Online]. Available: http://dx.doi.org/10.1137/040621697

[9] K. Astrom and B. Bernhardsson, "Comparison of riemann and lebesgue sampling for first order stochastic systems," in Decision and Control, 2002, Proceedings of the 41st IEEE Conference on, vol. 2, dec. 2002, pp. $2011-2016$.

[10] K. J. Åström, "Event based control," in Analysis and Design of Nonlinear Control Systems: In Honor of Alberto Isidori. Springer Verlag, 2007.

[11] J. Lunze and D. Lehmann, "A state-feedback approach to event-based control," Automatica, vol. 46, no. 1, pp. 211 - 215, 2010. [Online]. Available: http://www.sciencedirect.com/ science/article/pii/S0005109809004968

[12] P. Tabuada, "Event-triggered real-time scheduling of stabilizing control tasks," Automatic Control, IEEE Transactions on, vol. 52, no. 9, pp. $1680-1685$, sept. 2007.

[13] J. Pearson, J. P. Hespanha, and D. Liberzon, "Control with minimum communication cost per symbol," in Proc. of the 53nd Conf. on Decision and Contr., Dec. 2014, pp. 6050-6055.

[14] E. Kofman and J. Braslavsky, "Level crossing sampling in feedback stabilization under data-rate constraints," in Decision and Control, 2006 45th IEEE Conference on, Dec 2006, pp. 4423-4428.

[15] D. Lehmann and J. Lunze, "Event-based control using quantized state information," in Estimation and Control of Networked Systems, 2010, pp. 1-6.

[16] P. Tallapragada and N. Chopra, "On co-design of event trigger and quantizer for emulation based control," in American Control Conference (ACC), 2012, June 2012, pp. 3772-3777.

[17] R. Goebel, R. Sanfelice, and A. Teel, Hybrid Dynamical Systems: Modeling, Stability, and Robustness. Princeton University Press, 2012. [Online]. Available: http://books.google.com/books?id=L8qqFWBt3L8C

[18] L. Li, X. Wang, and M. Lemmon, "Stabilizing bitrates in quantized event triggered control systems," in Proceedings of the 15th ACM international conference on Hybrid Systems: Computation and Control. ACM, 2012, pp. 245-254.

[19] P. Tallapragada and J. Cortes, "Event-triggered stabilization of linear systems under bounded bit rates," ArXiv e-prints, May 2014.

[20] S. C. Tatikonda, "Control under communication constraints," Ph.D. dissertation, Massachusetts Institute of Technology, 2000. [Online]. Available: http://hdl. handle.net/1721.1/16755 